\title{
Effect of [EMIM]Ac Recycling on Salix gracilistyla Miq. Pretreatment for Enzymatic Saccharification ${ }^{1}$
}

\author{
Song-Yi HAN ${ }^{2}$ - Chan-Woo $\mathrm{PARK}^{2,3} \cdot$ Gu-Joong $\mathrm{KWON}^{2,4} \cdot$ Jong-Ho $\mathrm{KIM}^{2,3}$ • \\ Nam-Hun $\mathrm{KIM}^{2,3} \cdot$ Seung-Hwan LEE (iD) ${ }^{2,3,5, \dagger}$
}

\begin{abstract}
Recycling of ionic liquid (1-ethyl-3-methylimidazolium acetate, [EMIM]Ac) after the pretreatment of Salix gracilistyla Miq. was conducted and the effect of the recycling number on the enzymatic saccharification yield was investigated. Enzymatic saccharification was performed using an enzyme cocktail (Acremonium cellulase and Optimash BG) at $50{ }^{\circ} \mathrm{C}$ for $72 \mathrm{~h}$. All recycled [EMIM]Ac samples showed a lower amount of water soluble fraction than pure [EMIM]Ac. On increasing the recycling number from 1 to 4 , the amount of water soluble fraction decreased from $18 \%$ to $15 \%$. The X-ray diffraction pattern of the products pretreated with recycled [EMIM]Ac showed cellulose I crystalline polymorph. The crystallinity of the product pretreated with recycled [EMIM]Ac was 47-49\%, which was lower than $33 \%$ of that with pure [EMIM]Ac. The yields of glucose and xylose decreased in the pretreatment with recycled [EMIM]Ac compared to that with pure [EMIM]Ac.
\end{abstract}

Keywords: ionic liquid, recycling, pretreatment, enzymatic saccharification, Salix gracilistyla Miq.

\section{INTRODUCTION}

Pretreatment is an essential process to improve the enzymatic saccharification efficiency of lignocellulosic biomass because of its recalcitrance characteristics $(\mathrm{Wu}$ et al., 2013). Moreover, pretreatment is the most expensive process, which reportedly costs approximately $20 \%$ of the total cost for ethanol production from lignocellulosic biomass (Yang and Wyman 2008). In this context, many studies have attempted to develop a novel pretreatment process to improve the economic feasi- bility of the biorefinery process from lignocellulosic biomass (Jung et al., 2017; Yanti et al., 2019). Among them, the pretreatment using ionic liquids (ILs) has attracted attention as an environment-friendly process because of their various advantages such as low volatility, high thermal and chemical stability, excellent solubility, non-flammability, and recovery. IL pretreatments are also known effective as the biological and chemical conversion of lignocellulosic biomass into a wide range of biomaterials and bioenergy (Park et al., 2015; Dutta et al., 2016; Han et al., 2020c). The IL

${ }^{1}$ Date Received February 17, 2020, Date Accepted May 1, 2020

2 Institute of Forest Science, Kangwon National University, Chuncheon 24341, Republic of Korea

${ }^{3}$ Department of Forest Biomaterials and Engineering, College of Forest and Environmental Sciences, Kangwon National University, Chuncheon 24341, Republic of Korea

${ }^{4}$ Kangwon Institute of Inclusive Technology, Kangwon National University, Chuncheon 24341, Republic of Korea

${ }^{5}$ Institute of Fermentation and Brewing, Kangwon National University, Chuncheon 24341, Republic of Korea

† Corresponding author: Seung-Hwan LEE (e-mail: 1shyhk@kangwon.ac.kr, ORCID: 0000-0002-9988-2749) 
pretreatment is known to be more environment-friendly and facile but less economical than other organic-solvent-based processes (Kim et al., 2012; George et al., 2015). Therefore, it is recommended to recover and reuse the ILs for industrial applications (Elgharbawy et al., 2016). Sangian et al. (2015) investigated the effect of recycling an IL (methylmethylimidazolium dimethyl phosphate, [MMIM]DMP) after pretreatment of coconut coir for enzymatic saccharification. The used IL was recycled twice to re-employ for pretreatment. The yields of sugar after the pretreatment using fresh IL and IL recycled once and twice were $0.19,0.15$, and $0.15 \mathrm{~g}$ per gram of polysaccharide (cellulose+hemicellulose), respectively. Ding et al. (2016) pretreated corn stover using dilute $\mathrm{NaOH}$ ( $1 \mathrm{wt} \%$ solution) followed by pretreatment with fresh and recycled IL (1-butyl-3-methylimidazolium chloride, [BMIM]Cl). The glucose concentration from the product pretreated with the IL recycled 10 times was $18.7 \mathrm{~g} / \mathrm{L}$ after $12 \mathrm{~h}$ of enzymatic hydrolysis, which was $5.5 \mathrm{~g} / \mathrm{L}$ lower than that with fresh IL. Cheenkachorn et al. (2016) reported that the pretreatment of rice straw with [EMIM]Ac could afford the glucose yield of $90 \%$ compared to untreated products. The recycling of [EMIM]Ac using methanol as an anti-solvent reportedly retains more than $90 \%$ efficiency without any modification in the pretreatment process despite 5 recycles. Xu et al. (2017) investigated the effect of recycling 1-allyl-3-methylimidazolium chloride ([AMIM]Cl) and 1-butyl-3-methylimidazolium acetate ([BMIM]Ac) on the pretreatment of eucalyptus for enzymatic hydrolysis. The glucose yield by ILs recycled 4 times was $54.3 \%$ for [AMIM]Cl and $72.8 \%$ for [BMIM]Ac, which was 5.0- and 6.7-folds higher than that of untreated eucalyptus. The pretreatment effect of the recycled ILs deteriorated after 4 times, showing relatively lower sugar conversion and lignin removal.

In this study, the effect of the number of recycling of [EMIM]Ac on the enzymatic saccharification was investigated to improve the economic feasibility of the pretreatment. The higher solid loading of $15 \mathrm{wt} \%$ was adapted, which is higher than about 5\% in the general studies described above. Salix gracilistyla Miq. is a native species in Korea and known as one of the rapidly growing wood species for bioenergy production (Lee et al., 2018).

\section{MATERIALS and METHODS}

\subsection{Materials}

Salix gracilistyla Miq. wood powder (40 mesh) was extracted at $85{ }^{\circ} \mathrm{C}$ for $5 \mathrm{~h}$ with an ethanol/benzene mixed solution $(1: 2, \mathrm{v} / \mathrm{v})$ and used as the lignocellulose material. The chemical composition of Salix gracilistyla Miq. was 47.2, 26.4, 25.1, and $5.6 \mathrm{wt} \% \alpha$-cellulose, hemicellulose, klason lignin, and extractives, respectively and these results have been reported previously (Han et al., 2017a). [EMIM]Ac was purchased from IoLiTec (Heilbronn, Germany), and other chemicals used in this study were obtained from Daejung Chemicals \& Metals (Gyeonggi, Korea). Acremonium cellulase and Optimash BG were purchased from Meiji Seika Co. (Tokyo, Japan) and Genencor International, Inc. (Palo Alto, CA, USA).

\subsection{Pretreatment and IL re-usage}

The Salix gracilistyla Miq. wood powder was pretreated using [EMIM]Ac with $15 \mathrm{wt} \%$ solid loading at $120{ }^{\circ} \mathrm{C}$ for $2 \mathrm{~h}$ (Han et al., 2017b; Han et al., 2020a). The pretreated product was regenerated using $500 \mathrm{~mL}$ distilled water at room temperature for $1 \mathrm{~h}$. The regenerated Salix gracilistyla Miq. was collected by filtration under vacuum and washed with distilled water (500 $\mathrm{mL})$ to remove the residual $\mathrm{IL}$ and water soluble fraction. The fractionated residue was freeze-dried using FDB5503 (Operon Co., Ltd., Korea) and subjected to crystallinity analysis and enzymatic saccharification. The filtrate was collected from recycling the IL with an evaporator (Rotavapor, R-3000; Buchi Analytical Inc., USA) 
Effect of [EMIM]Ac Recycling on Salix gracilistyla Miq. Pretreatment for Enzymatic Saccharification

at $45{ }^{\circ} \mathrm{C}$ under a pressure of $700 \mathrm{mmHg}$ and stored at $40{ }^{\circ} \mathrm{C}$ for 3 days, which was sufficient to remove almost all the water from the IL. [EMIM]Ac was recycled for 1-4 times. Then, the recycled IL was used as the raw material, and the treatment procedure was the same as mentioned above.

\subsection{Acid hydrolysis}

The compositional measurement of the monosaccharides before and after pretreatment was conducted using the Laboratory Analytical Procedure (LAP) by NREL (Sluiter et al. 2008). The raw material and pretreated samples $(100 \mathrm{mg})$ were added to $1.5 \mathrm{~mL}$ Eppendorf tubes with $72 \%$ sulfuric acid solution $(1 \mathrm{~mL})$. For hydrolysis, the tubes were kept in a shaking incubator (Shaking incubator, VS-101Si, vision scientific Co., Korea) at $30{ }^{\circ} \mathrm{C}$ for $1 \mathrm{~h}$. The hydrolysates were diluted to $4 \%$ using deionized water $(19.8 \mathrm{~mL})$ and further hydrolyzed at $120{ }^{\circ} \mathrm{C}$ for $1 \mathrm{~h}$. Next, it was separated from the acid slurry for the measurement of acid-insoluble lignin to determine the acid-soluble monosaccharide. Calcium carbonate was used to neutralize the sample to a $\mathrm{pH}$ of 5-6. The supernatant was passed through a $0.2 \mu \mathrm{m}$ filter into an autosampler vial before monosaccharide analysis by HPLC. The yields of glucose and xylose were measured by HPLC (YL9100, Younglin Co., Anyang, Korea) with a carbohydrate analysis column (Aminex HPX-87P, 300×7.8 mm, hydrogen form, 8\% cross-linkage, Bio-Rad Laboratories, Inc., CA, USA) at a flow rate $0.6 \mathrm{~mL} / \mathrm{min}$ at $85{ }^{\circ} \mathrm{C}$. For acid-insoluble lignin measurement, the acid slurry was filtrated through the Whatman filter paper by adding distilled water until neutralized, dried at $100{ }^{\circ} \mathrm{C}$, and weighed.

\subsection{Enzymatic saccharification}

Enzymatic hydrolysis was conducted using an enzyme cocktail of Acremonium cellulase (15 FPU per gram of substrate) and Optimash BG $(0.2 \%, v / v)$ as a supplement to $\beta$-xylosidase (Han et al., 2020b). The pretreated substrates $(250 \mathrm{mg})$ were added to the enzyme cocktail at a solid loading of $2.5 \%$ in a sodium acetate buffer solution (50 mM, pH 5). Hydrolysis was performed in a shaking incubator at $50{ }^{\circ} \mathrm{C}$ for $72 \mathrm{~h}(150$ $\mathrm{rpm})$. Hydrolysates of $1 \mathrm{~mL}$ were sampled at $3,6,12$, 24, 48, and $72 \mathrm{~h}$ intervals. The enzyme inactivation was conducted by heating at $95{ }^{\circ} \mathrm{C}$ for $15 \mathrm{~min}$ in a heating block (HB-48-Set, Wisd; Daihan Scientific Co., Ltd., Wonju, Korea) and stored at $4{ }^{\circ} \mathrm{C}$. The enzymatic hydrolysate was centrifuged at $7000 \mathrm{rpm}$ for $15 \mathrm{~min}$ and filtered through a syringe filter $(0.2 \mu \mathrm{m})$.

\subsection{Analysis}

X-ray diffraction analysis was conducted for the crystallization evaluation of the pretreated sample. For this analysis, the specimen was prepared by substituting the suspension with t-butyl alcohol and freeze-drying. X-ray diffraction analysis was performed at a rate of $2^{\circ}$ per minute using an X-ray diffractometer (Rigaku RINTTTR III, Tokyo, Japan) at a range of $2 \theta=2-60^{\circ}$. The crystallinity index (CrI) was determined by the Segal method (Segal et al., 1959).

The monosaccharide analysis was conducted with an HPLC system (YL9100; Young Lin Instrument Co., Ltd., Anyang, Korea) equipped with an Aminex HPX87P column (Bio-Rad Laboratories, Inc., Hercules, CA, USA). The mobile phase was deionized water, and the flow rate was $0.6 \mathrm{~mL} / \mathrm{min}$ at $85{ }^{\circ} \mathrm{C}$. The glucose and xylose yields in the enzymatic hydrolysate were calculated using equation 1 and 2 :

Glucose or xylose yield (\%) $=\frac{\text { Mass of glucose or xylose in enzymatic hydrolysates }(\mathrm{g}) \times \text { conversion factors }}{\text { Mass of glucan or xylan in the pretreated wood }(\mathrm{g})} \times 100$

Glucose or xylose yield (\%)

$=\frac{\text { Mass of glucose or xylose in enzymatic hydrolysates }(\mathrm{g})}{\text { Mass of raw material }(\mathrm{g})} \times 100$ 
where 0.9 and 0.88 are conversion factors for glucose and xylose to glucan and xylan, respectively.

\section{RESULTS and DISCUSSION}

Fig. 1 shows the effect of the recycling number of [EMIM]Ac on the amount of water soluble fraction. Compared to the $21 \%$ fraction obtained by pure [EMIM]Ac, all samples pretreated with recycled [EMIM]Ac showed lower values. On increasing the recycling number from 1 to 4 , the amount of water soluble fraction decreased from $18 \%$ to $15 \%$. The water soluble fraction may contain low molecules of hemicellulose and lignin degraded by [EMIM]Ac, which can act as a physical barrier to block enzyme accessibility to cellulose (Shi et al., 2014; Kumagai et al., 2016). After extracting the water soluble fraction, the residual substrate will be cellulose-rich and very porous, which will increase the surface area of cellulose. Lee et al. (2010) reported that the nanospace could be generated between cellulose microfibrils by extracting hemicellulose by the hot-compressed water treatment. Moreover, delignification can increase the surface area of polysaccharides, i.e., cellulose and hemicellulose.

The main aim of pretreatment is to disrupt the cell wall structure of the lignocellulosic biomass by partial or complete removal of hemicellulose or lignin, resulting in the increase in the surface area and porosity of the substrates, and to decrease cellulose crystallinity (Kucharska et al., 2018). Fig. 2 shows the XRD patterns of the products pretreated with pure and recycled [EMIM]Ac for different recycling numbers, and their $\mathrm{CrI}$ are summarized in Table 1 . The raw material and the product pretreated with pure and recycled [EMIM]Ac displayed a strong peak at around $22.8^{\circ}$ $(2 \theta)$, corresponding to the (002) crystal plane of cellulose, as well as composite peaks at $15^{\circ}$ and $15.5^{\circ}$, corresponding to the (101) and (10) crystal planes. This pattern represents the cellulose I polymorph. In the sample pretreated with pure [EMIM]Ac, however, the

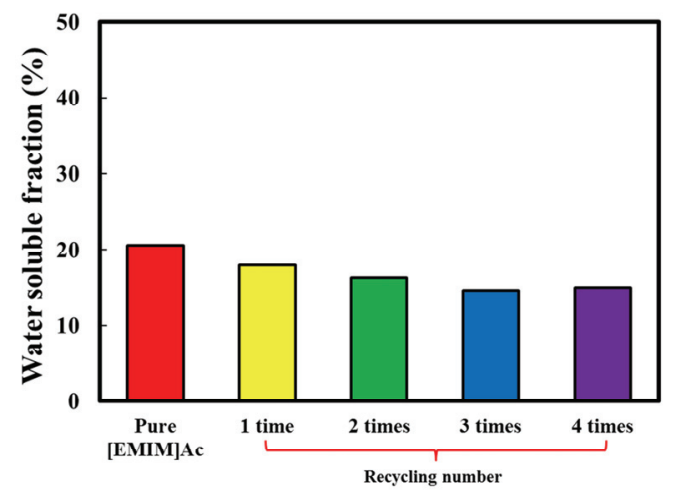

Fig. 1. Effect of the recycling number of [EMIM]Ac on water soluble fraction from Salix gracilistyla Miq.

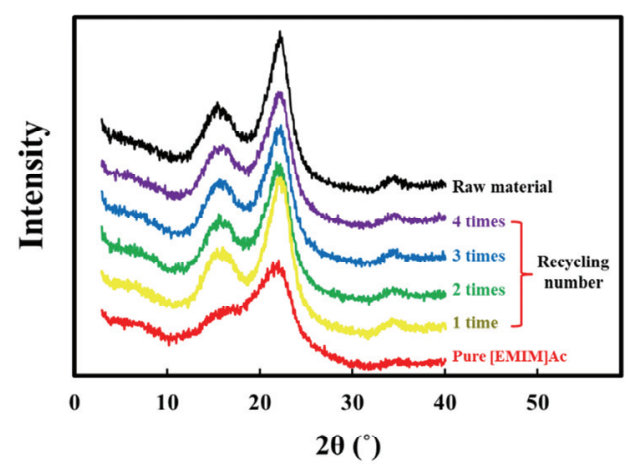

Fig. 2. XRD patterns of the products pretreated with pure and recycled [EMIM]Ac for different recycling numbers.

Table 1. Recycling numbers of [EMIM]Ac and CrI of the pretreated products

\begin{tabular}{ccccccc} 
& \multirow{2}{*}{ Raw material } & Pure & \multicolumn{4}{c}{ Number of the recycling } \\
\cline { 3 - 7 } & & [EMIM]Ac & 1 & 2 & 3 & 4 \\
\hline \hline CrI $(\%)$ & 55 & 33 & 49 & 47 & 47 & 47 \\
\hline
\end{tabular}


intensity of the (002) plane dramatically decreased and no distinct peak for the (101) and (10) planes were observed. This indicates that the crystallinity of cellulose is significantly disrupted by partial dissolution and distension of the cellulose crystal (Zhang et al., 2014). The increase in the amorphous portion of cellulose will improve its enzyme accessibility (Ling et al., 2017). This disruption ability was diminished by [EMIM] Ac recycling, regardless of the recycling numbers.

The CrI of the raw material was 55\%, which decreased to $33 \%$ by pretreatment with pure [EMIM]Ac. However, the CrI of the product pretreated with recycled [EMIM]Ac ranged from 47 to $49 \%$, which was higher than that with pure [EMIM]Ac. This indicates that the pretreatment effect of lowering the $\mathrm{CrI}$ is deteriorated by recycling. Hamidah et al. (2018) reported the pretreatment of softwood (Cryptomeria japonica) with a recycled [EMIM]Ac without the dissolution of cellulose and lignin. It was reported that $\mathrm{CrI}(34.0 \%)$ of the product pretreated with [EMIM]Ac recycled for 3 times were similar with $30.7 \%$ of the products with pure [EMIM]Ac, and glucose yields were also not significantly different between the products pretreated with recycled and pure [EMIM]Ac. Although lignin dissolved in [EMIM]Ac continued to accumulate after [EMIM]Ac recycling, the pretreatment was found to be effective for three consecutive cycles without the need for the removal of dissolved materials.

Fig. 3 shows the glucose and xylose yields of the product pretreated with pure and recycled [EMIM]Ac depending on enzymatic saccharification time. The glucose and xylose yields were evaluated from the original masses of glucan and xylan, which were obtained by acid hydrolysis. With increasing enzymatic saccharification time, the glucose and xylose yield increased in all products. The product pretreated with pure [EMIM]Ac showed the highest glucose and xylose yields, which were more than $80 \%$ for $72 \mathrm{~h}$ saccharification. On the other hand, the raw material exhibited the lowest yields, i.e., approximately $20 \%$ and $10 \%$ for glucose and xylose, respectively. With recycled [EMIM]Ac, both yields decreased compared to pure [EMIM]Ac. There was no significant difference in both yields among the products pretreated with recycled [EMIM]Ac yields of $66-69 \%$ for glucose and $55-63 \%$ for xylose for $72 \mathrm{~h}$ saccharification.

The glucose and xylose yields of the product pretreated with pure and recycled [EMIM]Ac after enzymatic saccharification are calculated using equation 2 and summarized in Table 2. These values are calculated on the basis of the original mass and the glucan (xylan) after acid hydrolysis (equation 1) in paren
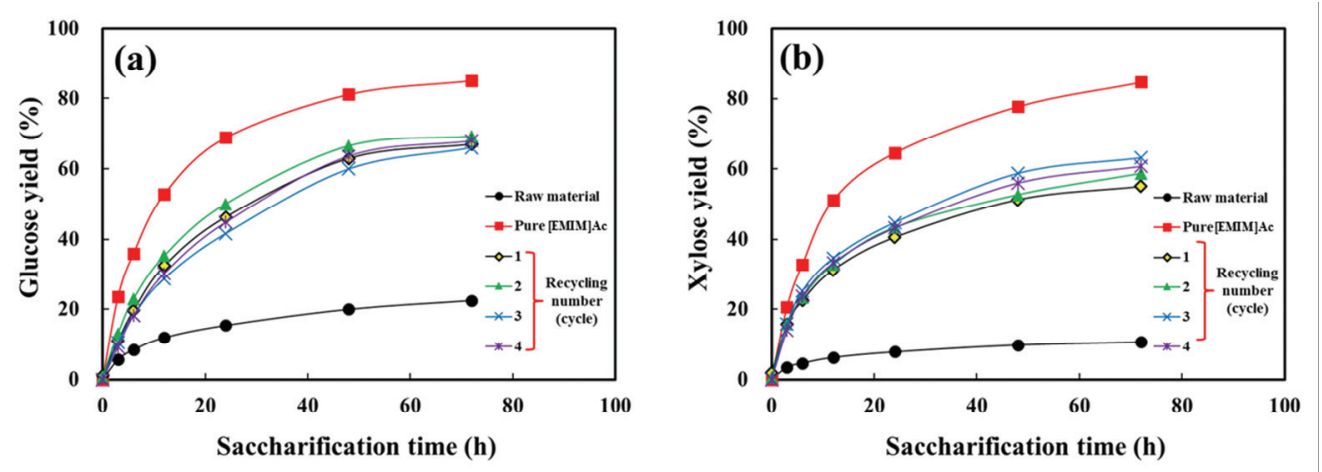

Fig. 3. Yields of glucose (a) and xylose (b) for raw material and product pretreated with pure and recycled [EMIM]Ac on the basis of glucan and xylan obtained by acid hydrolysis. 
Table 2. Yields of glucose and xylose for pretreated products on the basis of the original mass after enzymatic saccharification for $72 \mathrm{~h}$

\begin{tabular}{|c|c|c|c|}
\hline & & Yield of enzymatic & saccharification \\
\hline & & Glucose $(\%)$ & Xylose (\%) \\
\hline $\begin{array}{c}\text { Raw } \\
\text { material }\end{array}$ & & $14(22)$ & $3(11)$ \\
\hline Pure IL & & $42(85)$ & $13(85)$ \\
\hline \multirow{4}{*}{$\begin{array}{l}\text { Recycling } \\
\text { number }\end{array}$} & 1 & $34(67)$ & $9(55)$ \\
\hline & 2 & $32(69)$ & $10(59)$ \\
\hline & 3 & $34(66)$ & $11(63)$ \\
\hline & 4 & $34(68)$ & $12(61)$ \\
\hline
\end{tabular}

Notes: The values in parenthesis are calculated on the basis of the mass of glucan and xylan after enzymatic saccharification for $72 \mathrm{~h}$.

thesis as well. The glucose and xylose yields of the product pretreated with pure [EMIM]Ac were $42 \%$ and $13 \%$, respectively. These values decreased with the pretreatment with recycled [EMIM]Ac. Regardless of the recycling number, the glucose yield on enzymatic saccharification was $34-36 \%$, which was lower than that with pure [EMIM]Ac. However, the xylose yield $(9-12 \%)$ was not significantly different from that with pure [EMIM]Ac (13\%). This result is attributed to the extraction amount of the water soluble fraction because the value was calculated by compensating the amount of water soluble fraction. This also indirectly indicates that the water soluble fraction contains hemicellulose components degraded by [EMIM]Ac.

In general, there is a correlation between the saccharification yield and the CrI of the cellulose (Cui et al., 2014). It is also known that the initial rate of the cellulase-catalyzed hydrolysis of cellulose is linearly proportional to the crystallinity index of cellulose. In this study, the CrI of the product pretreated with pure [EMIM]Ac was 33\%, showing the highest saccharification yield. On the other hand, the CrI values (47-49\%) increased for the product pretreated with recycled [EMIM]Ac, showing the lower saccharifi- cation yield than that with pure [EMIM]Ac. Hall et al. (2010) reported that the CrI of cellulose is a key predictor of the enzymatic saccharification efficiency and rate. They stated that the initial enzymatic rate continued to increase with a decreasing crystallinity index, even though the bound enzyme concentration stayed constant. They insisted that their finding supports the determinant role of crystallinity rather than adsorption on the enzymatic rate. Hou et al. (2012) reported that $[\mathrm{Ch}]$ Lys showed excellent reusability over five successive batches in the pretreatment of rice straw. Lignin continued to accumulate in [Ch]Lys during recycling, and delignification efficiency decreased slightly from $59 \%$ to $53 \%$ over the five recycling batches for rice straw. Glucose yields $(84-87 \%)$ and initial glucose release rates $(2.4-2.5 \mathrm{mg} / \mathrm{mL} / \mathrm{h})$ remained almost constant after the IL was reused for five batches.

\section{CONCLUSION}

Pretreatment with recycled [EMIM]Ac of Salix gracilistyla Miq. was successfully conducted to achieve more than $80 \%$ yield of glucose and xylose by enzymatic saccharification. Compared to the product pretreated with pure [EMIM]Ac, the product pretreated with recycled [EMIM]Ac showed a lower amount of water soluble fraction, cellulose I polymorph, and higher CrI, and lower yields of glucose and xylose. However, there was no significant difference in these properties among the product pretreated with recycled [EMIM]Ac. It is concluded that the pretreatment effect of recycled [EMIM]Ac was declined, compared to pure [EMIM]Ac, but more than $60 \%$ enzymatic saccharification yield was able to be achieved by the pretreatment using [EMIM]Ac recycled for 4 times. 


\section{ACKNOWLEDGMENT}

This study was funded by the Basic Science Research Program through the National Research Foundation of Korea (NRF) funded by the Ministry of Education (Grant No. 2018R1A6A1A03025582).

\section{REFERENCES}

Cheenkachorn, K., Douzou, T., Roddecha, S., Tantayotai, P., Tantayotai, P., Sriariyanun, M. 2016. Enzymatic saccharification of rice straw under influence of recycled ionic liquid pretreatments. Energy Procedia 100: 160-165.

Cui, T., Li, J., Yan, Z., Yu, M., Li, S. 2014. The correlation between the enzymatic saccharification and the multidimensional structure of cellulose changed by different pretreatments. Biotechnology for Biofuels 7: 134.

Ding, J.C., Xu, G.C., Han, R.Z., Ni, Y. 2016. Biobutanol production from corn stover hydrolysate pretreated with recycled ionic liquid by Clostridium saccharobutylicum DSM 13864. Bioresource Technology 199: 228-234.

Dutta, T., Shi, J., Sun, J., Zhang, X., Cheng, G., Simmons, B.A., Singh, S. 2016. Ionic liquid pretreatment of lignocellulosic biomass for biofuels and chemicals. In: Ionic liquids in the biorefinery concept: challenges and perspectives. RSC Green Chemistry Ser. 36, Ed. by Bogel-Lukasik, R., Royal Society of Chemistry, Cambridge, UK.

Elgharbawy, A.A., Alam, M.Z., Moniruzzaman, M., Goto, M. 2016. Ionic liquid pretreatment as emerging approaches for enhanced enzymatic hydrolysis of lignocellulosic biomass. Biochemical Engineering Journal 109: 252-267.

George, A., Brandt, A., Tran, K., Zahari, S.M.S.N.S., Klein-Marcuschamer, D., Sun, N., Sathitsuksanoh, N., Shi, J., Stavila, V., Parthasarathi, R., Singh,
S., Holmes, B.M., Welton, T., Simmons, B.A., Hallett, J.P. 2015. Design of low-cost ionic liquids for lignocellulosic biomass pretreatment. Green Chemistry 17(3): 1728-1734.

Hall, M., Bansal, P., Lee, J.H., Realff, M.J., Bommarius A.S. 2010. Cellulose crystallinity-a key predictor of the enzymatic hydrolysis rate. The FEBS Journal 277(6): 1571-1582.

Hamidah, U., Arakawa, T., H'ng, Y.Y., Nakagawaizumi, A., Kishino, M. 2018. Recycled ionic liquid 1-ethyl-3-methylimidazolium acetate pretreatment for enhancing enzymatic saccharification of softwood without cellulose regeneration. Journal of Wood Science 64(2): 149-156.

Han, S.Y., Park, C.W., Kim, N.H., Lee, S.H. 2017a. Co-solvent system of [EMIM]Ac and DMF to improve the enzymatic saccharification of pussy willow (Salix gracilistyla Miq.). Holzforschung 71(1): 43-50.

Han, S.Y., Park, C.W., Lee, S.H. 2017b. Preparation of lignocellulose nanofiber by mechanical defibrillation after pretreatment using cosolvent of ionic liquid and DMF. Journal of the Korean Wood Science and Technology 45(3):268-277.

Han, S.Y., Park, C.W., Febrianto, F., Kim, N.H., Lee, S.H. 2020a. Pretreatment with [EMIM]Ac/DMAc Co-solvent to Improve Enzymatic Saccharification of Pussy Willow (Salix gracilistyla Miq.). BioResources 15(1): 187-198.

Han, S.Y., Park, C.W., Endo, T., Febrianto, F., Kim, N.H., Lee, S.H. 2020b. Extrusion process to enhance the pretreatment effect of ionic liquid for improving enzymatic hydrolysis of lignocellulosic biomass. Wood Science and Technology.

Han, S.Y., Park, C.W., Park, J.B., Ha, S.J., Kim, N.H., Lee, S.H. 2020c. Ethanol fermentation of the enzymatic hydrolysates from the products pretreated using [EMIM]Ac and its co-solvents with DMF. Journal of Forest and Environmental Science 36(1): 
Song-Yi HAN • Chan-Woo PARK • Gu-Joong KWON $\cdot$ Jong-Ho KIM • Nam-Hun KIM $\cdot$ Seung-Hwan LEE

62-66.

Hou, X.D., Smith, T.J., Li, N., Zong, M.H. 2012. Novel renewable ionic liquids as highly effective solvents for pretreatment of rice straw biomass by selective removal of lignin. Biotechnology and Bioengineering 109(10): 2484-2493.

Jung, J.Y., Ha, S.Y., Park, J.H., Yang, J.-K. 2017. Optimization of alkali pretreatment from steam exploded barley husk to enhance glucose fraction using response surface methodology. Journal of the Korean Wood Science and Technology 45(2): 182-194.

Kim, J.-Y., Kim, T.-S., Hwang, H., Oh, S., Choi, J.W. 2012. Chemical - Structural characterization of lignin extracted from Pitch Pine with Ionic Liquid (1-ethyl-3-methylimidazolium acetate). Journal of the Korean Wood Science and Technology 40(3): 194-203

Kucharska, K., Rybarczyk, P., Holowacz, I., Glinka, M., Kaminski, M. 2018. Pretreatment of lignocellulosic materials as substrates for fermentation processes. Molecules 23(11): 2937.

Kumagai, A., Lee, S.H., Endo, T. 2016. Evaluation of the effect of hot-compressed water treatment on enzymatic hydrolysis of lignocellulosic nanofibrils with different lignin content using a quartz crystal microbalance. Biotechnology and Bioengineering 113(7): 1441-1447.

Lee, S.H., Chang, F., Inoue, S., Endo, T. 2010. Increase in enzyme accessibility by generation of nanospace in cell wall supramolecular structure. Bioresource Technology 101(19): 7218-7223.

Lee, H.S., An, C.H., Kang, J.W., Lee, W.Y., Yi, J.-S. 2018. Investigation of Growth Characteristics of Salix gracilistyla Clones. Journal of Korean Society of Forest Science 107(1): 16-24.

Ling, Z., Chen, S., Zhang, X., Takabe, K., Xu, F. 2017. Unraveling variations of crystalline cellulose induced by ionic liquid and their effects on enzymatic hydrolysis. Scientific Reports 7: 10230.

Park, S.Y., Hwang, H., Jang, S., Choi, I.G., Choi, J. 2015. study on pretreatment of giant miscanthus using ionic liquid and structural change of lignin. Journal of the Korean Wood Science and Technology 43(3): 344-354.

Sangian, H., Kristian, J., Rahma, S., Dewi, H.K., Puspasari, D.A., Agnesty, S.Y., Gunawan, S., Widjaja, A. 2015. Preparation of reducing sugar hydrolyzed from high-lignin coconut coir dust pretreated by the recycled ionic liquid [mmim][dmp] and combination with alkaline. Bulletin of Chemical Reaction Engineering \& Catalysis 10(1): $8-22$.

Segal, L., Creely, J.J., Martin Jr, A.E., Conrad, C.M. 1959. An empirical method for estimating the degree of crystallinity of native cellulose using the X-ray diffractometer. Textile Research Journals 29(10): 786-794.

Shi, J., Balamurugan, K., Parthasarathi, R., Sathitsuksanoh, N., Zhang, S., Stavila, V., Zhang, S., Stavila, V., Subramanian, V., Simmons, B.A., Singh, S. 2014. Understanding the role of water during ionic liquid pretreatment of lignocellulose: Co-solvent or anti-solvent. Green Chemistry 16(8): 3830-3840.

Sluiter, A., Crocker, D., Hames, B., Ruiz, R., Scarlata, C., Sluiter, J., Templeton, D. 2008. Determination of structural carbohydrates and lignin in biomass," NREL/TP-510-42618.

Wu, L., Lee, S.H., Endo, T. 2013. Effect of dimethyl sulfoxide on ionic liquid 1-ethyl-3-methylimidazolium acetate pretreatment of eucalyptus wood for enzymatic hydrolysis. Bioresource Technology 140: 90-96.

Xu, J., Liu, B., Hou, H., Hu, J. 2017. Pretreatment of eucalyptus with recycled ionic liquids for low-cost biorefinery. Bioresource Technology 234: 406-414. 
Yang, B., Wyman, C.E. 2008. Pretreatment: the key to unlocking low-cost cellulosic ethanol. Biofuels, Bioproducts and Biorefining 2(1): 26-40.

Yanti, H., Syafii, W., Wistara, N.J., Febrianto, F., Kim, N.H. 2019. Effect of Biological and Liquid Hot Water Pretreatments on Ethanol Yield from Mengkuang (Pandanus artocarpus Griff). Journal of the Korean Wood Science and Technology 47(2): 145-162.

Zhang, J., Wang, Y., Zhang, L., Zhang, R., Liu, G., Cheng, G. 2014. Understanding changes in cellulose crystalline structure of lignocellulosic biomass during ionic liquid pretreatment by XRD. Bioresource Technology 151: 402-405. 